$900 \mathrm{~cm}^{-1}$ ). Single element Attenuated total reflectance (ATR) contains averaged FTIR spectra from the superficial 10 microns of an entire oesophageal biopsy. We aim to extract individual cellular characteristics with molecular resolution by using FTIR and ATR on ex-vivo oesophageal biopsy specimens from patients undergoing endoscopy for BE surveillance or neoplasia assessment to detect high grade dysplasia (HGD).

Methods 731 spectra of 374 fresh biopsies from 76 patients were analysed. Biopsies were taken from visible BE. Before being placed in formalin, they were analysed by a spectrometer fitted with liquid nitrogen-cooled detector and ATR silicon microprism. For each spectrum 500 interferograms were averaged before Fourier transformation. Spectra were pre-processed using MATLAB scripts by spectrally removing liquid water and water vapour contributions, vector normalising to the $1610-900 \mathrm{~cm}^{-1}$ region and second derivative conversion to remove baseline artefacts. Specific cellular characteristics were first determined. Unstained $8 \mu \mathrm{m}$ tissue sections from 1 patient were analysed with FPA (Focal Plane Array)-FTIR imaging and correlated with stained slides. It was possible to accurately describe specific features ofsquamous epithelium (SQ), columnar lined epithelium (CLE), and lamina propria (LP) with this method. These features were applied to the 374 fresh biopsies using ATR-FTIR. Combined clustering and partial least squares regression discrimination (PLSDA) was used to build a diagnostic pipeline. Biopsies were grouped according to their cellular characteristics from the prior FTIR imaging. (1. SQ vs Rest, 2. SQ only biopsies, 3. CLE only biopsies, 4. CLE and LP containing biopsies and 5. LP containing biopsies only).

Results We distinguished SQ mucosa from CLE (BE), HGD and OAC tissue at an overall sensitivity of $89 \%$ and specificity of $91 \%$. By grouping the spectra into groups according to their cellular contents, HGD was distinguished from all other biopsies with sensitivities and specificities of 68 and 89\% (CLE only), 74 and 82\% (CLE and LP) and 94 and 97\% (LP only) respectively. Conclusion Combined FTIR and ATR-FTIR spectroscopy can accurately distinguish HGD arising in BE on ex-vivo biopsy specimens and might become accurate enough to exclude routine histopathological evaluation in these patients.

Disclosure of Interest None Declared.

\section{OC-016 THE TOLL-LIKE RECEPTOR PATHWAY IS RECURRENTLY MUTATED IN OESOPHAGEAL ADENOCARCINOMA}

${ }^{1}$ DR Fels Elliottt ${ }^{*}{ }^{1}$ CS Ross-Innes, ${ }^{2} \mathrm{MD}$ Eldridge, ${ }^{1} \mathrm{RC}$ Fitzgerald on behalf of OCCAMS oesophageal International Cancer Genome Consortium. 'Hutchison/MRC Cancer Unit, University of Cambridge, Cambridge, UK; ${ }^{2}$ Bioinformatics Core, Cancer Research UK Cambridge Institute, Cambridge, UK

\subsection{6/gutjnl-2014-307263.16}

Introduction The interaction between the oesophageal microbiota and the inflammatory microenvironment in Barrett's carcinogenesis is poorly understood. One of the mechanisms by which microbiota may induce chronic inflammation is by triggering Toll-like receptor (TLR) signalling and activation of nuclear factor kappa B. We aimed to utilise whole genome sequencing (WGS) data to investigate TLR mutations and expression in oesophageal adenocarcinoma (OAC), with a focus on TLR9.

Methods We interrogated the mutational profiles of 66 OAC samples, with matched germline references from each case, which had undergone WGS as part of the oesophageal ICGC study. All mutations were verified using PCR and Sanger sequencing. To further explore TLR9 expression along the
Barrett's progression sequence, we performed TLR9 immunohistochemistry on tissue microarray samples including normal squamous oesophagus $(\mathrm{N}=16)$, duodenum $(\mathrm{N}=14)$, non-dysplastic Barrett's $(\mathrm{N}=53)$, low-grade dysplasia $(\mathrm{N}=13)$, high-grade dysplasia $(\mathrm{N}=25)$ and OAC $(\mathrm{N}=338)$. Within the large cohort of OAC samples we binarised the intensity scores $(0-1$ and $2-3$ ) and examined whether there were any significant differences in relation to clinicopathologic variables (TNM stage, histological grade, lymphovascular invasion, survival).

Results We identified missense mutations in TLR pathway genes in 8/66 (12.1\%) of OAC samples, including TLR1 (1.5\%), TLR4 (3\%), TLR7 (1.5\%), TLR9 (3\%), MYD88 (1.5\%), and TRAF6 $(1.5 \%)$. TLR9 protein was expressed more highly in Barrett's and OAC than normal oesophageal squamous tissue $(\mathrm{p}<$ 0.001). The expression in Barrett's was similar to duodenum, however immunopositivity was increased in OAC $(\mathrm{p}<0.05)$ compared with this control tissue. The staining intensity was generally consistent throughout the Barrett's progression sequence with strong immunopositivity (intensity score 3) in $7.7-14.5 \%$ of samples. Within the OAC cohort, there was no significant association between TLR9 expression and any of the clinicopathological variables tested. The only significant difference in survival was observed in a small subset of patients with metastatic disease ( $\mathrm{N}=14$ patients), where median survival was significantly decreased for patients with TLR9 intensity score 23 (8 months \pm 2.24 (standard error)) compared to patients with TLR9 intensity score $0-1$ (18 months \pm 6.57$), \mathrm{p}<0.05$.

Conclusion TLR pathway genes appear to be recurrently mutated in OAC, which given the mutational context and heterogeneity of disease ${ }^{1}$ could represent significant involvement of the TLR signalling pathway in Barrett's carcinogenesis.

\section{REFERENCE}

1 Dulak AM, et al. Exome and whole-genome sequencing of esophageal adenocarcinoma identifies recurrent driver events and mutational complexity. Nat Genet. 2013 May; 45(5):478-86

Disclosure of Interest None Declared.

\section{OC-017 AUTOFLUORESCENCE-TARGETED OPTICAL BIOPSY ACCURATELY DIAGNOSES DYSPLASIA IN BARRETT'S OESOPHAGUS AND CAN DETECT THE FIELD OF MOLECULAR CHANGE}

${ }^{1} \mathrm{M}$ Di Pietro*, ${ }^{2} \mathrm{E}$ Bird-Lieberman, ${ }^{1} \mathrm{X}$ Liu, ${ }^{3} \mathrm{M}$ O'Donovan, ${ }^{1} \mathrm{R}$ Fitzgerald. ${ }^{1}$ Cancer Unit, Medical Research Council, Cambridge, UK; ${ }^{2}$ Oxford University Hospital NHS Trust, Oxford, UK; ${ }^{3}$ Histopathology, Cambridge University Hospital NHS Trust, Cambridge, UK

\subsection{6/gutjnl-2014-307263.17}

Introduction Probe-based confocal laser endomicroscopy (pCLE) allows optical biopsies in Barrett's oesophagus (BO) to predict histological outcome but it is subject to sampling error if performed in a random fashion. We used autofluorescence imaging (AFI) to direct pCLE and added molecular biomarkers to the histopathological diagnosis. The aims of this study were to assess the diagnostic accuracy for dysplasia of AFI-targeted optical biopsies and to investigate the correlation between pCLE patterns and field of molecular change.

Methods 46 patients with BO (non-dysplastic BE $n=20$, indefinite for dysplasia $n=4$, low grade dysplasia $n=10$, high grade dysplasia (HGD) or intramucosal cancer $(\mathrm{IMC}) \mathrm{n}=12$ ) were recruited at a single centre. Patients underwent high-resolution endoscopy followed by AFI and then pCLE was performed on AFI positive (AFI+) areas. Targeted biopsies were taken from 